\title{
Neuroadrenergic disarray in pseudo-resistant and resistant hypertension
}

\author{
Guido Grassi ${ }^{1,2}$, Michele Bombelli ${ }^{1}$, Silvia Buzzi ${ }^{1}$, Marco Volpe ${ }^{1}$ and Gianmaria Brambilla ${ }^{1}$ \\ Several studies have investigated the behavior of sympathetic cardiovascular drive in essential hypertension, providing \\ conclusive evidence of the adrenergic activation characterizing this condition. These studies have also shown the importance \\ of neuroadrenergic overdrive in the development and progression of the hypertensive state as well as in the pathogenesis of \\ hypertension-related end-organ damage. The information available on the sympathetic nervous system's behavior in 'pseudo- \\ resistant' and 'true resistant' hypertension is much more scarce. This paper will review the available knowledge on this issue \\ by examining the data collected via indirect and direct approaches to investigate adrenergic function in resistant hypertension \\ as well as the effects of pharmacological and non-pharmacological interventions.
}

Hypertension Research (2014) 37, 479-483; doi:10.1038/hr.2014.25; published online 27 February 2014

Keywords: ambulatory blood pressure monitoring; office blood pressure; resistant hypertension; sympathetic nervous system; white-coat hypertension

\section{INTRODUCTION}

Cumulative evidence collected over the past few decades strongly supports the notion that homeostatic control of the cardiovascular system exerted by the sympathetic nervous system undergoes marked alterations in a consistent fraction of hypertensive patients. These alterations, represented by a marked increase in the adrenergic nervous system's excitatory influences on the heart as well as on the peripheral circulation, are detectable in the earlier stages of hypertensive disease. ${ }^{1,2}$ As hypertension becomes more stable, however, the sympathetic abnormalities undergo further potentiation, thereby contributing directly and indirectly to the maintenance of blood pressure elevation, disease progression and the development of target organ damage. ${ }^{1,2}$ An additional step in the complex chain of events leading to the development and progression of adrenergic abnormalities relates to the evidence that several major pathological states ascribed to cardiovascular (heart failure), metabolic (diabetes mellitus, obesity, metabolic syndrome, sleep apnea) and renal (renal insufficiency and failure) factors often accompany and complicate chronic blood pressure elevation and may further aggravate the above-mentioned neurogenic alterations. ${ }^{1-6}$ In some of these conditions (that is, congestive heart failure, renal failure and stroke), evidence suggests that sympathetic activation may have independent prognostic relevance, with the magnitude of the adrenergic overdrive being inversely related to patient survival. ${ }^{1-2,4}$

There is little information available on the sympathetic nervous system's behavior in resistant hypertension, that is, the clinical condition characterized by elevated blood pressure despite the use of three or more antihypertensive drugs, including a diuretic at a full daily dosage. This lack of information may depend on several factors, including difficulties in diagnosing 'true' resistant hypertension as well as the methodological problems related to direct assessment of sympathetic neural drive in this condition.

This study will first provide an overview of the neuroadrenergic abnormalities characterizing the hypertensive state and the abnormalities' possible determinants. This will be followed by an analysis of the information available on the behavior of direct and indirect adrenergic drive markers in 'pseudo-resistant' and 'true resistant' hypertension. Finally, the implications of these findings for resistant hypertension treatment will be briefly mentioned.

\section{SYMPATHETIC ABNORMALITIES IN EARLY AND ADVANCED HYPERTENSIVE STAGES}

Early clinical stages of hypertensive disease (and in some cases, prehypertensive stages, particularly in subjects with a family history of hypertension) are characterized by the so-called hyperkinetic circulatory state, which is mediated both by increased adrenergic drive and reduced parasympathetic function. ${ }^{7}$ Such reciprocal changes in autonomic cardiovascular modulation have been documented by several studies, which can be summarized as follows.

In young, borderline hypertensive subjects, i.v. administration of atropine (which blocks the effects of the parasympathetic neurotransmitter acetylcholine on muscarinic receptors) triggers a lower increase in heart rate and cardiac output than that reported in pure normotensive age-matched controls. ${ }^{7}$ This alteration, which

${ }^{1}$ Clinica Medica, Dipartimento di Medicina Clinica, Prevenzione e Biotecnologie Sanitarie, Università Milano-Bicocca, Monza, Italy and ${ }^{2}$ RCCS Multimedica, Sesto San Giovanni, Milan, Italy

Correspondence: Professor G Grassi, Clinica Medica, University of Milano-Bicocca, Ospedale San Gerardo dei Tintori, Via Pergolesi 33, 20052 Monza (Milano), Italy. E-mail: guido.grassi@unimib.it

Received 28 March 2013; revised 4 July 2013; accepted 14 September 2013; published online 27 February 2014 
demonstrates the impairment of vagal-heart rate control that occurs in hypertension, is not limited to the parasympathetic function, but affects sympathetic cardiovascular control as well.

Manifold evidence supports this statement. In a meta-analysis of all published studies on indirect sympathetic tone markers, such as plasma norepinephrine, these markers were significantly elevated in essential hypertensive patients compared with age-matched normotensive subjects. ${ }^{8}$ Furthermore, by employing a technique based on i.v. tracer infusion of small radiolabelled norepinephrine doses, Australian investigators were able to show that the rate of norepinephrine spillover from neuroeffector junctions is increased in young subjects with borderline blood pressure elevation and that this process takes place in the kidney and in the heart, that is, two organs important to homeostatic blood pressure control. ${ }^{9}$ Further evidence comes from the direct measurement of sympathetic nerve traffic to the skeletal muscle circulation, a technique which has documented increased central sympathetic outflow in young, borderline hypertensive subjects. ${ }^{1,2,4,6}$

Complex borderline hypertension syndrome, however, is characterized by other abnormalities involving the hemodynamic state, the metabolic and hormonal profile as well as hemorheological conditions. Several of these abnormalities are triggered and reinforced by autonomic abnormalities, specifically by sympathetic overdrive. This appears to be particularly the case for the metabolic disarray, which is frequently detected in early hypertensive phases and includes hyperinsulinemia, insulin resistance, dyslipidemia and hypercholesterolemia. ${ }^{6}$ Most of these alterations, which represent the main features of metabolic syndrome together with visceral obesity, are characterized by marked adrenergic overdrive, as studies based on direct recording of muscle sympathetic neural outflow and the norepinephrine spillover technique have unequivocally shown. , $2,4,6^{-1}$

The above-mentioned sympathetic abnormalities reported in the early stages of hypertension appear to be maintained and potentiated in established hypertension. This outcome has been shown by a study performed by our group, ${ }^{10}$ in which we quantified sympathetic nerve traffic to the skeletal muscle district in three groups of age-matched subjects, that is, those with normal blood pressure, moderate essential hypertension and more severe essential hypertension. The progressive increase in blood pressure values observed in these three clinical conditions was paralleled by a progressive and marked elevation in sympathetic nerve traffic, suggesting adrenergic overdrive's key role not only in the development but also in the progression of the hypertensive state. A few other issues related to the autonomic alterations that characterize essential hypertension deserve to be mentioned.

First, sympathetic overactivity is not only a feature of young and middle-age hypertensives but also occurs in elderly hypertensives, even when systolic blood pressure values are selectively elevated. ${ }^{11,12}$ Indeed, when sympathetic nerve traffic was recorded in elderly subjects with systodiastolic or isolated systolic hypertension, a clearcut sympathetic activation was observed when the values were compared with those found in healthy, elderly, normotensive controls. ${ }^{12}$ Second, the hypertension-related increase in adrenergic outflow appears to be specific to some cardiovascular districts, such as the heart, kidney and skeletal muscle vasculature, and almost peculiar to the essential hypertensive state. ${ }^{2,10,12-14}$ Finally, the adrenergic overdrive appears to be related to some extent to the 24-h absolute blood pressure load as well as the day/night blood pressure difference. ${ }^{15}$ This finding is supported by the evidence provided by our group and others that hypertensive patients with the 'reverse dipping profile' (that is, patients in whom blood pressure values do not undergo any reduction overnight but rather show a tendency to increase), are characterized by more pronounced sympathetic activation than that seen in dipper hypertensives. ${ }^{16}$

\section{BEHAVIOR OF SYMPATHETIC CARDIOVASCULAR DRIVE IN 'PSEUDO-RESISTANT' HYPERTENSION}

A consistent fraction of hypertensive patients labeled as resistant hypertensives are indeed 'pseudo-resistant.' In an analysis, we recently performed of the results collected in two large observational studies carried out and coordinated by our group, the Pressioni Arteriose Monitorate E loro Associazioni (PAMELA) and the Blood Pressure (BP) control rate and CArdiovascular Risk profilE (BP-CARE) study, we found that $\sim 1 / 3$ of the patients originally diagnosed with resistant hypertension are indeed characterized by white-coat hypertension when ambulatory blood pressure monitoring is performed and/or home blood pressure measurements are taken into account. ${ }^{17,18}$ Assessment of sympathetic neural drive in white-coat hypertension has been performed by different investigators, and the results are quite different from those collected in true resistant hypertension patients.

All the approaches employed to test adrenergic function (power spectral analysis of the heart rate signal, plasma norepinephrine concentrations in peripheral blood and direct recording of efferent postganglionic sympathetic nerve traffic in a peripheral nerve) show that white-coat hypertension, which is responsible for a consistent number of pseudo-resistant hypertensive states, is characterized by adrenergic overdrive. ${ }^{19-21}$ The magnitude of this activation, however, does not appear to be different from that seen in essential hypertensive states in which both 'office' and 'out-of-office' blood pressure values are elevated.

This finding was shown years ago by the results of a study we performed in white-coat and essential hypertensives, who were classified by stringent criteria, including ambulatory blood pressure values. ${ }^{21}$ As shown in Figure 1, muscle sympathetic nerve traffic, both when expressed as burst incidence over time and when corrected for heart rate values, was elevated in white-coat hypertensive patients compared with true normotensive individuals. However, when the values were compared with those obtained in true essential hypertensive patients, it was clear that the white-coat group showed a lower degree of adrenergic activation (Figure 1). ${ }^{21}$ As discussed below, neuroadrenergic function in white-coat and 'pseudo-resistant' hypertension is different from that displayed by 'true' resistant hypertensive patients. In these patients, a potentiation of the sympathetic overdrive, which characterizes essential hypertension, can be detected via the microneurographic nerve traffic recording technique. $^{22}$

It can thus be concluded that 'pseudo-resistant' hypertension differs from 'true' resistant hypertension not only from a hemodynamic view point, that is, the difference between office and out-ofoffice blood pressure values, but also based on the pathophysiological background (Table 1). As discussed below, resistant hypertension displays more pronounced activation of sympathetic neural influences on the cardiovascular system compared with 'pseudo-resistant' hypertension.

\section{SYMPATHETIC FUNCTION IN 'TRUE RESISTANT' HYPERTENSION}

Scanty information is available on sympathetic cardiovascular function in resistant hypertension. Among the likely factors responsible for this lack of data, two appear to have a major role. The first one refers to the already mentioned difficulty in correctly diagnosing 'true' resistant hypertension, accounting for the white-coat effect as well as 

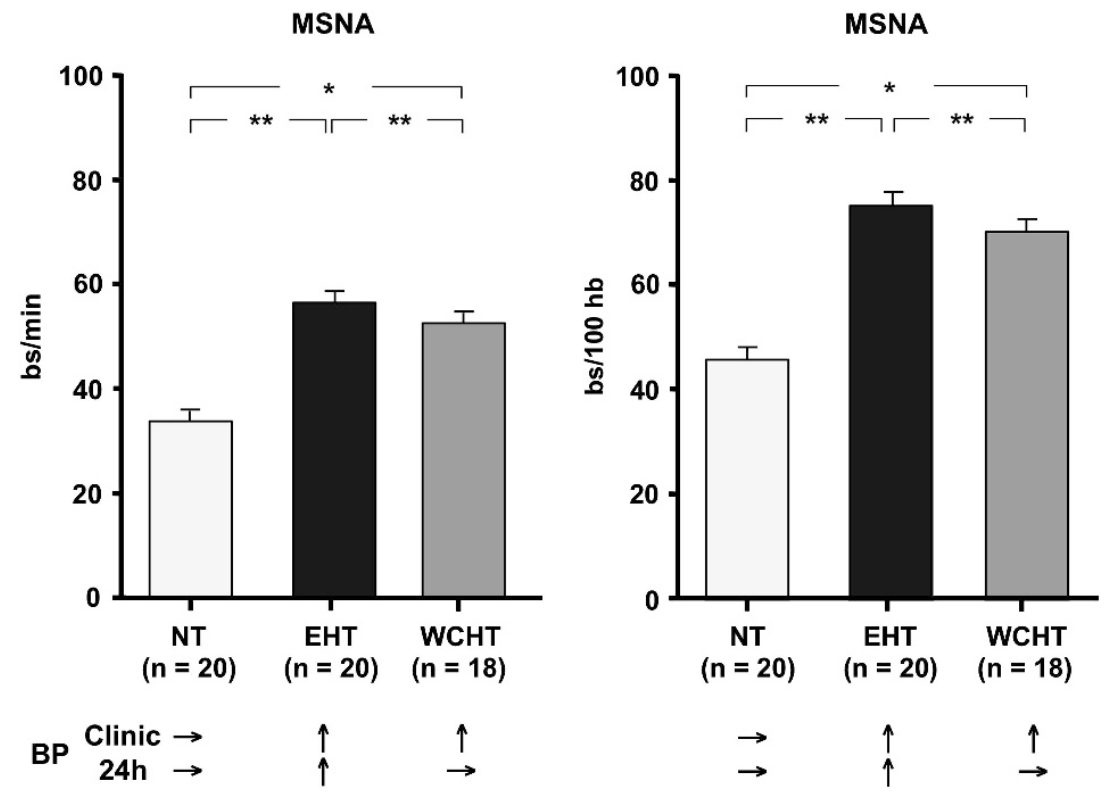

Figure 1 Muscle sympathetic nerve traffic values (MSNA), expressed as burst incidence over time (bs min ${ }^{-1}$, left panel) and as burst incidence corrected for heart rate (bs per $100 \mathrm{hb}$, right panel) in normotensive subjects (NT, normal clinical and ambulatory blood pressure), essential hypertensive patients (EHT, elevated clinical and ambulatory blood pressure) and white-coat hypertensive patients (WCHT, elevated clinical but normal blood pressure). Data are shown as the mean \pm s.e. Asterisks $\left({ }^{*} P<0.05,{ }^{*} P<0.01\right)$ refer to the statistically significant differences between groups. BP: blood pressure. Arrows indicate the normality $(\rightarrow)$ or elevation $(\uparrow)$ of clinical and ambulatory $(24 \mathrm{~h})$ blood pressure in the 3 groups of subjects. Figure created using data from Grassi et al. ${ }^{21}$

Table 1 Blood pressure and sympathetic proflles of pseudo-resistant and resistant HT

\begin{tabular}{lll}
\hline & Pseudo-resistant HT & Resistant HT \\
\hline Office BP values & Increased & Marked increased \\
Home BP values & Normal & Increased \\
Ambulatory BP values & Normal & Increased \\
Sympathetic nervous system & Moderately activated & Markedly activated
\end{tabular}

Abbreviations: BP, blood pressure; HT, hypertension.

poor patient adherence to prescribed antihypertensive drug regimens. However, the second factor refers to the complicated antihypertensive drug treatment schedules present in resistant hypertensive patients, which affect sympathetic neural function assessments.

Despite these caveats and limitations, in the past few years, some indirect and direct data have been collected on the neuroadrenergic profile of resistant hypertensive patients. The first data set is based on an analysis of regional norepinephrine spillover, obtained via the previously mentioned norepinephrine radiolabelled technique. ${ }^{9}$ In 10 patients with resistant hypertension who underwent renal denervation, Esler and coworkers and Schlaich and coworkers reported data related to renal norepinephrine spillover, showing a consistent reduction (on average 47\%) of this direct marker of sympathetic renal function 30 days after radiofrequency ablation of the renal nerves. ${ }^{23-25}$ Baseline renal norepinephrine spillover data, assessed by the authors before the renal nerve ablation procedure, however, may explain, although indirectly, whether and to what extent the sympathetic nervous system is activated in resistant hypertension.

When we compare the data of the renal norepinephrine spillover reported in these patients with those observed in a previous study carried out by the same authors in essential non-resistant hypertensive patients, ${ }^{26}$ the values tend to be greater in the resistant hypertensive group. This is particularly the case when the data are analyzed for the age range between 60 and 79 years, which is a common age for detecting resistant hypertensives. Although these data need to be interpreted with some caution, given the 'historical' nature of the control group, they may indirectly suggest the presence of high adrenergic overdrive in these patients.

More direct information on the patterns of the sympathetic neural function in resistant hypertension come from data collected in a series of studies carried out by our group through the PAMELA study, with the aim to assess indirectly or directly the behavior of the adrenergic neural influences on the heart and peripheral circulation in this hypertensive state. In the first set of studies, we examined the two cyclic components of systolic and diastolic blood pressure residual variability identified via a fast Fourier transform spectral analysis of 24-h ambulatory blood pressure tracings. ${ }^{27}$

The first of these two components has been shown to be closely related to sympathetic nerve traffic values, for both systolic and diastolic blood pressure values. ${ }^{27,28}$ When this analysis was applied to a selected group of hypertensive patients from the PAMELA study, we found that this first component was significantly greater in the case of systolic blood pressure in resistant hypertensives compared with the values seen in controlled and uncontrolled hypertensives (Figure 2). This was also the case for the second cyclic component and the residual variability values for systolic but not for diastolic blood pressure (Figures 2 and 3).

Taken together, these data support the hypothesis that sympathetic cardiovascular influences undergo greater potentiation in resistant hypertension than that characterizing non-resistant hypertension. Recently, this hypothesis has received further experimental support from the finding that sympathetic nerve traffic values are significantly greater in resistant than in non-resistant hypertensive patients with similar levels of blood pressure elevation. ${ }^{29}$ 

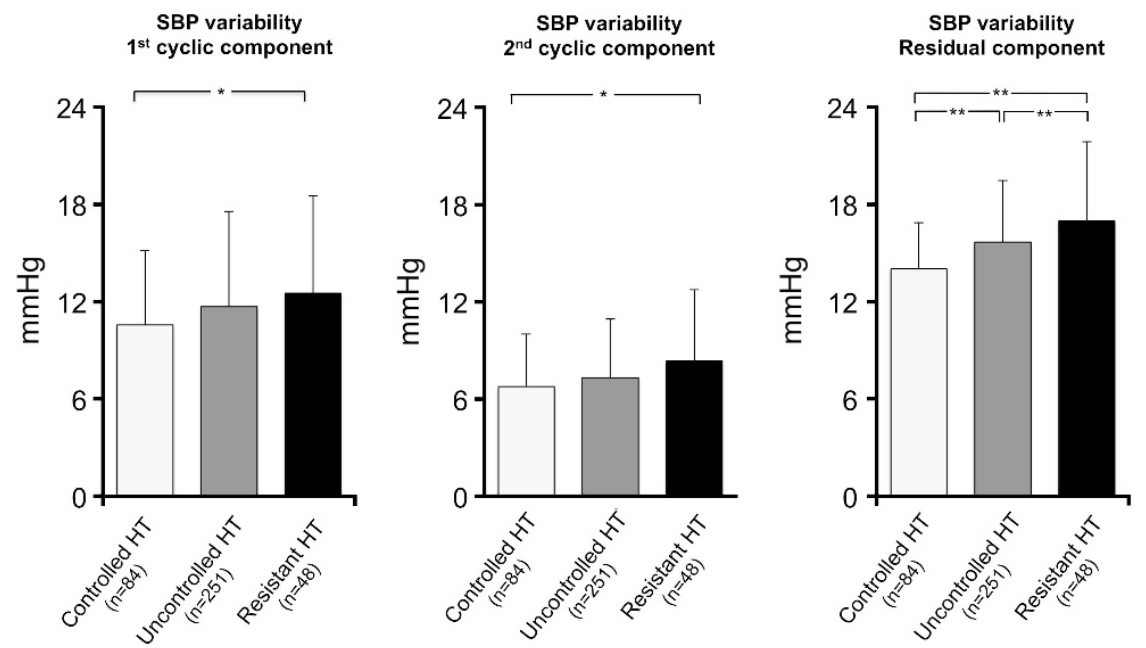

Figure 2 Behavior of the cyclic and residual components of systolic blood pressure (SBP) variability in controlled, uncontrolled and resistant hypertensive (HT) patients of the Pressioni Arteriose Monitorate E loro Associazioni study. Data are shown as the mean \pm s.e. Asterisks $\left({ }^{*} P<0.05,{ }^{* *} P<0.01\right)$ refer to the statistically significant differences between groups.

DBP variability
$1^{\text {st }}$ cyclic componen

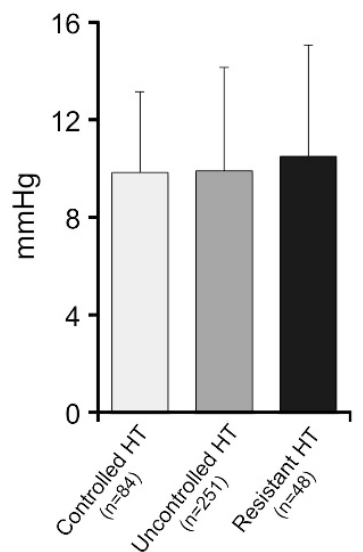

DBP variability

$2^{\text {nd }}$ cyclic component

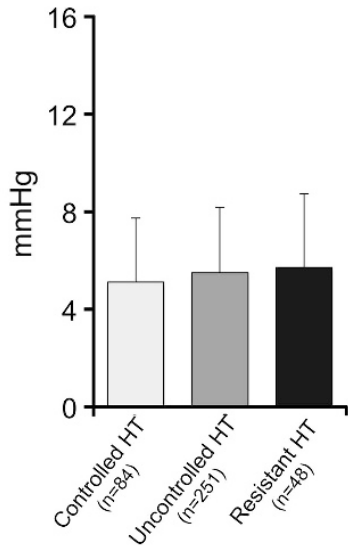

DBP variability

Residual component

Figure 3 Behavior of the cyclic and residual components of diastolic blood pressure (DBP) variability in controlled, uncontrolled and resistant hypertensive (HT) patients of the diastolic blood pressure study. Data are shown as the mean \pm s.e. Values are not significantly different between the three study groups.

Two further issues related to the neuroadrenergic abnormalities characterizing resistant hypertension deserve to be mentioned. The first abnormality refers to the evidence that a consistent fraction of hypertensive patients resistant to antihypertensive drug treatment also displays sleep apnea syndrome. ${ }^{30}$ This condition, characterized by a marked elevation in sympathetic nerve traffic values in both obese and lean subjects, ${ }^{31,32}$ may participate in the profound sympathoexcitation occurring in the resistant hypertensive state. The second abnormality refers to the finding that an aldosterone excess has been repeatedly reported in these patients. ${ }^{30,33}$ Taking into account the sympathoexcitatory effects of this hormone, ${ }^{13,34}$ this pathway may represent another pathophysiological mechanism that determines the adrenergic overdrive seen in these patients.

\section{THERAPEUTIC IMPLICATIONS}

The evidence described in the previous sections represents the background for considering sympathetic deactivation as an important therapeutic goal of non-pharmacological and pharmacological interventions for resistant hypertension treatment. This is the case, for example, for antialdosterone drugs, such as spironolactone, whose administration has been shown to reduce norepinephrine spillover rate as well as sympathetic neural outflow. ${ }^{35,36}$ This is also the case for electrical stimulation of carotid sinus baroreceptors, which has been shown to consistently reduce sympathetic nerve traffic values and concomitantly elevated blood pressure values. ${ }^{37}$ Finally, this is the case for radiofrequency ablation of renal nerves, a procedure capable of exerting marked sympathoinhibitory effects in resistant hypertension, as confirmed by the reduction in renal and total norepinephrine spillover rate as well as in muscle sympathetic nerve traffic observed a few weeks after the renal denervation procedure. ${ }^{23,24,37}$ However, further studies are warranted to better define the time course of the sympathoinhibitory effects of the renal denervation approach, their qualitative and quantitative relationships with the blood-pressurelowering responses and their potential impact on clinical outcomes. 


\section{CONFLICT OF INTEREST}

The authors declare no conflict of interest.

1 Esler MD. The sympathetic nervous system through the ages: from Thomas Willis to resistant hypertension. Exp Physiol 2011; 96: 611-622.

2 Grassi G, Bertoli S, Seravalle G. Sympathetic nervous system. Role in hypertension and in chronic kidney disease. Curr Opin Nephrol Hypertens 2012; 21: 46-51.

3 Bradley TD, Floras JS. Obstructive sleep apnoea and its cardiovascular consequences. Lancet 2009; 373: 82-93.

4 Malpas SC. Sympathetic nervous system overactivity and its role in the development of cardiovascular disease. Physiol Rev 2010; 90: 561-57.

5 Abboud FM. The Walter B. Cannon Memorial Award Lecture, 2009. Physiology in perspective: the wisdom of the body. In search of autonomic balance: the good, the bad and the ugly. Am J Physiol 2010; 298: R1449-R1467.

6 Grassi G. Sympathetic overdrive and cardiovascular risk in the metabolic syndrome. Hypertens Res 2006; 29: 839-847.

7 Julius S, Pascual AV, London R. Role of parasympathetic inhibition in the hyperkinetic type of borderline hypertension. Circulation 1971; 44: 413-418.

8 Goldstein DS. Plasma catecholamines and essential hypertension. An analytical review. Hypertension 1983; 5: 86-99.

9 Esler M, Jennings G, Lambert G, Meredith I, Horne M, Eisenhofer G. Overflow of catecholamine neurotransmitters to the circulation: source, fate and function. Physiol Rev 1990; 70: 963-985.

10 Grassi G, Cattaneo BM, Seravalle G, Lanfranchi A, Mancia G. Baroreflex control of sympathetic nerve activity in essential and secondary hypertension. Hypertension 1998; 81: 68-72.

11 Yamada Y, Miyajima E, Tochikubo O, Matsukawa T, Ishii M. Age-related changes in muscle sympathetic nerve activity in essential hypertension. Hypertension 1989; 13 (pt 2), 870-877.

12 Grassi G, Seravalle G, Bertinieri G, Turri C, Dell'Oro R, Stella ML, Mancia G. Sympathetic and reflex alterations in systo- diastolic and systolic hypertension of the elderly. J Hypertens 2000; 18: 587-593.

13 Miyajima E, Yamada Y, Yoshida Y, Matsukawa T, Shionoiri H, Tochikubo O, Ishii M. Muscle sympathetic nerve activity in renovascular hypertension and primary aldosteronism. Hypertension 1991; 17: 1057-1062.

14 Matsukawa T, Mano T, Gotoh E, Ishii M. Elevated sympathetic nerve activity in patients with acceperated essential hypertension. J clin Invest 1993; 92: 25-28.

15 Narkiewicz K, Winnicki M, Schroeder K, Phillips BG, Kato M, Cwalina E, Somers VK. Relationship between muscle sympathetic nerve activity and diurnal blood pressure profile. Hypertension 2002; 39: 168-172.

16 Grassi G, Seravalle G, Quarti-Trevano F, Dell'Oro R, Bombelli M, Cuspidi C, Facchetti R, Bolla G, Mancia G. Adrenergic, metabolic and reflex abnormalities in reverse and extreme dipper hypertensives. Hypertension 2008; 52: 925-931.

17 Bombelli M, Facchetti R, Brambilla G, Fodri D, Seravalle G, Peronio M, Nicoli F, Ganz F, Sega R, Grassi G, Mancia G. Clinic, home, 24-hour blood pressure profiles and clinical correlates in resistant hypertension: data from the PAMELA study. $J$ Hypertens 2012; 30: e53. (Abstract).

18 Brambilla G, Bombelli M, Seravalle G, Cifkova R, Laurent S, Narkiewicz K, Facchetti R, Redon J, Mancia G, Grassi G. Prevalence and clinical characteristics of patients with true resistant hypertension in central and eastern Europe: data from the BP-CARE study. J Hypertens 2013; 31: 2018-2024.

19 Neumann SA, Jennings JR, Muldoon MF, Manuck SB. White-coat hypertension and autonomic nervous system dysregulation. Am J Hypertens 2005; 18: 584-588.

20 Fagard RH, Stolarz K, Kuznetsova T, Seidlerova J, Tikhonoff V, Grodzicki T, Nikitin Y, Filipovsky J, Peleska J, Casiglia E, Thijs L, Staessen JA, Kawecka-Jaszcz K.
Sympathetic activity, as assessed by power spectral analysis of heart rate variability, in white-coat, masked and sustained hypertension versus true normotension. $J$ Hypertens 2007; 25: 2280-2285.

21 Grassi G, Seravalle G, Trevano FQ, Dell'oro R, Bolla G, Cuspidi C, Arenare F, Mancia G. Neurogenic abnormalities in masked hypertension. Hypertension 2007; 50: 537-542.

22 Seravalle G, Volpe M, Ganz F, Magni L, Brambilla G, Dell'Oro R, Bombelli M, Mancia G, Grassi G. Neuroadrenergic profile in patients with resistant hypertension. J Hypertens 2011; 29: e141 (Abstract).

23 Schlaich MP, Sobotka PA, Krum H, Lambert E, Esler MD. Renal sympathetic-nerve ablation for uncontrolled hypertension. New Engl J Med 2009; 361: 932-934.

24 Schlaich MP, Krum H, Whtibourn R, Walton T, Lambert GW, Sobotka PA, Esler MD. Effects of renal sympathetic denervation on noradrenaline spillover and systemic bood pressure in patients with resistant hypertension. J Hypertens 2009; 27: S154 (Abstract).

25 Esler M, Schlaich M, Sobotka P, Whitbourn R, Sadowski J, Bartus K, Kapelak B, Walton A, Sievert H, Thambar S, Abraha W, Krum H. Catheter-based renal denervation reduces total body and renal noradrenaline spillover and blood pressure in resistant hypertension. J Hypertens 2009; 27: S167 (Abstract).

26 Esler M, Lambert G, Jennings G. Regional norepinephrine turnover in human hypertension. Clin Exp Hypertens 1989; 11: S75-S89.

27 Dell'Oro R, Quarti F, Facchetti R, Seravalle G, Bombelli M, Polo Fritz H, Sega R, Grassi G, Mancia G. Overall, cyclic and non-cyclic 24-hour blood pressure variability and sympathetic nerve traffic in the elderly. J Hypertens 2003; 21: S213 (Abstract).

28 Mancia G, Bombelli M, Facchetti R, Madotto F, Corrao G, Trevano FQ, Grassi G, Sega R. Long-term prognostic value of blood pressure variability in the general population: results of the Pressioni Arteriose Monitorate e Loro Associazioni Study. Hypertension 2007; 49: 1265-1270.

29 Volpe M, Facchetti R, Magni L, Fodri D, Nicoli F, Mineo C, Scotton E, Giannattasio C, Bombelli M, Mancia G, Grassi G. Blood pressure variability in resistant hypertension: data from the PAMELA study. High Blood Press Cardiovasc Prev 2012; 19: 145. (Abstract).

30 Calhoun DA, Jones D, Textor S, Goff DC, Murphy TP, Toto RD, White A, Cushman WC White W, Sica D, Ferdinand K, Giles TD, Falkner B, Carey RMAmerican Heart Association Professional Education Committee. Resistant hypertension: diagnosis, evaluation, and treatment: a scientific statement from the American Heart Association Professional Education Committee of the Council for High Blood Pressure Research. Circulation 2008; 117: e510-e526.

31 Narkiewicz K, van de Borne PJ, Cooley RL, Dyken ME, Somers VK. Sympathetic activity in obese subjects with and without sleep apnea. Circulation 1998; 98: 772-776.

32 Grassi G, Facchini A, Trevano FQ, Dell'Oro R, Arenare F, Tana F, Bolla G, Monzani A, Robuschi M, Mancia G. Obstructive sleep apnea-dependent and -independent adrenergic activation in obesity. Hypertension 2005; 46: 321-325.

33 Gaddam KK, Nishizaka MK, Pratt-Ubunama MN, Pimenta E, Aban I, Oparil S, Calhoun DA. Characterization of resistant hypertension: association between resistant hypertension, aldosterone, and persistent intravascular volume expansion. Arch Intern Med 2008; 168: 1159-1164.

34 Gabor A, Leenen FH. Central neuromodulatory pathways regulating sympathetic activity in hypertension. J Appl Physiol 2012; 113: 1294-1303.

35 Wray DW, Supiano MA. Impact of aldosterone receptor blockade compared with thiazide therapy on sympathetic nervous system function in geriatric hypertension. Hypertension 2010; 55: 1217-1223.

36 Grassi G. Sympathetic neural activity in hypertension and related diseases. Am J Hypertens 2010; 23: 1052-1060.

37 Grassi G, Seravalle G, Brambilla G, Bombelli M, Dell'Oro R, Gronda E, Mancia G. Novel antihypertensive therapies: renal sympathetic nerve ablation and carotid baroreceptor stimulation. Curr Hypertens Rep 2012; 14: 567-572. 